EPJ Web of Conferences 32, 01003 (2012)

DOI: $10.1051 /$ epjconf/20123201003

(C) Owned by the authors, published by EDP Sciences, 2012

\title{
Scattering of ECRF waves by edge density blobs and fluctuations in tokamak plasmas
}

\author{
Abhay K. Ram ${ }^{1, a}$, Kyriakos Hizanidis ${ }^{2}$, and Yannis Kominis ${ }^{2}$ \\ 1 Plasma Science and Fusion Center, Massachusetts Institute of Technology, Cambridge, Massachusetts \\ 02139, USA. \\ 2 National Technical University of Athens (part of HELLAS), School of Electrical and Computer \\ Engineering, Association EURATOM-Hellenic Republic, GR-15773, Greece
}

\begin{abstract}
There are two basic approaches to studying the effects of density blobs and edge fluctuations on the coupling of electron cyclotron (EC) radio frequency waves to the core of tokamak plasmas. The first is the geometric optics approach in which the effect of fluctuations is to change the refractive properties of the EC beam or rays. There are two consequences of refractive scattering - diffusion in real space leading to a spatial deflection of the rays and diffusion in wave vector space leading to the broadening of the launched spectrum. The geometric optics approach is limited to small density fluctuations of $10 \%$ or less. The second approach to studying the effect of blobs on EC fields is using the full wave approach. This approach extends the range of validity well beyond that of geometric optics; however, it is theoretically and computationally much more challenging. In this paper a full wave model for scattering of radio frequency waves is developed. Results from the model demonstrate diffractive scattering of EC waves by density blobs and the enhancement of the electric fields near the surface of the blob.
\end{abstract}

\section{Introduction}

In fusion plasmas, radio frequency (RF) waves in the electron cyclotron (EC) and lower hybrid range of frequencies are commonly used to modify the current profile. In ITER, EC waves are expected to stabilize the neoclassical tearing mode by providing current in the island region. The RF waves propagate from the excitation structures to the core of the plasma through a dynamic edge region characterized by large density fluctuations and blobs $[1,2]$. These fluctuations can scatter RF waves through refraction and diffraction.

The usual treatment of refractive scattering is based on the geometric optics approximation. Using this approach we have developed a Fokker-Planck model for refractive scattering [3]. We find that the scattering from a random distribution of blobs can diffuse rays in configuration space and in wavevector space. The diffusion in space can make the rays miss their intended target region, while the diffusion in wave-vector space can broaden the wave spectrum. The latter can lead to modifications of the current profile and a lower current drive efficiency. The geometric optics approach is of limited validity as it is restricted by, at least, two aspects. It is not valid for density fluctuations that are $10 \%$ or more of the ambient density. From experimental observations it is evident that density fluctuations are generally much larger [4]. These large fluctuations not only lead to refraction but also to reflection of RF waves. Another limitation of geometric optics approximation is that it does not account for geometric differences between the phase front of the RF wave and the spatial profile of the fluctuations and blobs. In other words, geometric optics does not include the effect of diffraction.

In order to study the effect of large density fluctuations in the form of blobs, we have developed a a full-wave model for wave scattering which extends well beyond the limitations of geometric op-

\footnotetext{
a e-mail: abhay@mit.edu
}

This is an Open Access article distributed under the terms of the Creative Commons Attribution License 2.0, which permits unrestricted use, distribution, and reproduction in any medium, provided the original work is properly cited. 
tics. This model, a first of its kind study on scattering of plasma waves by blobs, includes diffractive scattering of waves as well as coupling of the incident wave to other propagating plasma waves. In particular, an incident ordinary wave in the EC frequency range can couple power to the extraordinary wave through scattering by blobs.

In the full wave theory, the vector Helmholtz equation is solved for the electromagnetic fields inside the blob and for the scattered fields outside the blob. The incident wave induces charge oscillations inside the blob leading to waves propagating in all directions inside the blob. The geometry of the blob manifests itself through the boundary conditions at the surface of the blob. The boundary conditions require the existence of scattered electromagnetic fields outside the blob. The combination of the incident wave and scattered fields has to match with the electromagnetic fields inside the blob through the appropriate boundary conditions satisfying Maxwell's equations. Our model leads to diffractive scattering and excitation of waves different from the incident wave. So the full wave treatment can lead to interesting modifications of the incoming EC wave.

From experimental observations it is found that the speed of propagations of the fluctuations and blobs is of the order of $5 \times 10^{3} \mathrm{~m} \mathrm{~s}^{-1}[2,4]$. This is slower than the group velocity of the incoming EC wave by several orders of magnitude. The frequency of temporal variation of the fluctuations is less than $1 \mathrm{MHz}$. So the EC frequency is several orders of magnitude higher than the fluctuation frequency. Consequently, in our model we will assume that the blobs are stationary. Observations also show that the scale lengths of the blobs typically range from about half a centimeter to several centimeters $[2,4]$.

In this paper we develop the analytical basis behind the full-wave scattering model and then apply it to the scattering of EC waves by a blob in a typical ITER-type edge plasma. The results in this paper are to demonstrate the physics that is inherent to full-wave scattering. More detailed numerical analysis will be forthcoming in other publications where detailed consequences of the full-wave scattering model will be discussed.

\section{Description of the geometry and the plasma}

We assume that the density of the background plasma and of the plasma in the blob are spatially uniform. The ratio of the density in the blob to the density of the background plasma is not constrained. The plasma is cold and the blob is a sphere of radius $a$. We use the spherical coordinate system with the origin being at the center of the blob. The position vector $\mathbf{r}$ is given by $(r, \theta, \phi)$ where $r$ is the radial distance, $\theta \in[0, \pi]$ is the polar angle, and $\phi \in[0,2 \pi]$ is the azimuthal angle. The propagation vector $\mathbf{k}$ is represented in its own spherical coordinate system by $\left(k, \theta^{\prime}, \phi^{\prime}\right)$. The ambient magnetic field in which the plasma and the blob are immersed is taken to be uniform and along the $z$ direction of the Cartesian coordinate system. The plasma is stationary and the electromagnetic RF fields have a time dependence of the form $\exp (-i \omega t)$ where $\omega$ is the angular frequency of the RF fields.

In the spherical coordinate system of the propagation vector $\mathbf{k}$, the plasma permittivity tensor is obtained by an appropriate transformation of the Cartesian permittivity tensor [5]. This transformed tensor is

$$
\stackrel{\leftrightarrow}{K}=\left(\begin{array}{ccc}
K_{\perp} \sin ^{2} \theta^{\prime}+K_{\|} \cos ^{2} \theta^{\prime} & \left(K_{\perp}-K_{\|}\right) \sin \theta^{\prime} \cos \theta^{\prime} & -i K_{x} \sin \theta^{\prime} \\
\left(K_{\perp}-K_{\|}\right) \sin \theta^{\prime} \cos \theta^{\prime} & K_{\perp} \cos ^{2} \theta^{\prime}+K_{\|} \sin ^{2} \theta^{\prime} & -i K_{x} \cos \theta^{\prime} \\
i K_{x} \sin \theta^{\prime} & i K_{x} \cos \theta^{\prime} & K_{\perp}
\end{array}\right)
$$


The elements of $\stackrel{\leftrightarrow}{K}$ are given by

$$
\begin{aligned}
& K_{\perp}=1-\frac{\omega_{p e}^{2}}{\omega^{2}-\omega_{c e}^{2}}-\sum_{l} \frac{\omega_{p i}^{2}}{\omega^{2}-\omega_{c i}^{2}} \\
& K_{x}=-\frac{\omega_{c e}}{\omega} \frac{\omega_{p e}^{2}}{\omega^{2}-\omega_{c e}^{2}}+\sum_{l} \frac{\omega_{c i}}{\omega} \frac{\omega_{p i}^{2}}{\omega^{2}-\omega_{c i}^{2}} \\
& K_{\|}=1-\frac{\omega_{p e}^{2}}{\omega^{2}}-\sum_{l} \frac{\omega_{p i}^{2}}{\omega^{2}}
\end{aligned}
$$

where $\omega_{p e}\left(\omega_{p i}\right)$ and $\omega_{c e}\left(\omega_{c i}\right)$ are the angular electron (ion) plasma frequency and cyclotron frequency, respectively, and the index $l$ represents all the ion species in the plasma. The permittivity tensor of the background plasma and of the blob are expressed in terms of their respective densities and ion compositions.

\section{Electric and magnetic fields}

The spatial form of the electric fields in a plasma is given by the Faraday-Ampere equation

$$
\nabla \times \nabla \times \mathbf{E}(\mathbf{r})=\frac{\omega^{2}}{c^{2}} \stackrel{\leftrightarrow}{K} \cdot \mathbf{E}(\mathbf{r})
$$

where $c$ is the speed of light. We solve for $\mathbf{E}(\mathbf{r})$ by using the Fourier transform

$$
\mathbf{E}(\mathbf{r})=\int d^{3} k \mathbf{E}(\mathbf{k}) e^{i \mathbf{k} \cdot \mathbf{r}}
$$

Substituting this form into (3) yields

$$
\int d^{3} k \stackrel{\leftrightarrow}{D} \cdot \mathbf{E}(\mathbf{k}) e^{i \mathbf{k} \cdot \mathbf{r}}=0
$$

where

$$
\stackrel{\leftrightarrow}{D}(\mathbf{k}, \omega)=\frac{c^{2}}{\omega^{2}}\left(\mathbf{k k}-k^{2} \stackrel{\leftrightarrow}{I}\right)+\stackrel{\leftrightarrow}{K}
$$

is the dielectric tensor. Here kk is a dyadic and $\overleftrightarrow{I}$ is the unit tensor. In general, the above equation is satisfied if

$$
\stackrel{\leftrightarrow}{D}(\mathbf{k}, \omega) . \mathbf{E}(\mathbf{k})=0
$$

For a non-trivial solution to the Fourier transformed electric field, we require that

$$
\operatorname{det}(\stackrel{\leftrightarrow}{D}(\mathbf{k}, \omega))=0
$$

where det denotes the determinant of the tensor. From (8) we obtain the dispersion relation relating the angle of propagation $\theta^{\prime}$ to the magnitude of $\mathbf{k}$

$$
\tan ^{2} \theta^{\prime}=\frac{-K_{\|}\left(n_{k}^{2}-K_{R}\right)\left(n_{k}^{2}-K_{L}\right)}{\left(n_{k}^{2}-K_{\|}\right)\left(K_{\perp} n_{k}^{2}-K_{R} K_{L}\right)}
$$

where $c k / \omega=n_{k}$ is the index of refraction, and $K_{R}=K_{\perp}+K_{x}$ and $K_{L}=K_{\perp}-K_{x}$. For a given angle of propagation $\theta^{\prime}$, there are two real roots for $n_{k}^{2}$ so that $n_{k}$ is either purely real or purely imaginary. Of 
the four roots for $n_{k}$ only two are independent. The other two can be obtained by appropriate rotations in $\theta^{\prime}$ and $\phi^{\prime}$.

From Eq. (7) we can evaluate the polarization of the electric field. For example, one possible set of polarizations is

$$
\begin{aligned}
& \frac{E_{\theta^{\prime}}}{E_{k}}=\frac{\bar{K} \sin \theta^{\prime} \cos \theta^{\prime}}{n_{k}^{2}-K_{\|}-\bar{K} \cos ^{2} \theta^{\prime}} \\
& \frac{E_{\phi^{\prime}}}{E_{k}}=\frac{i K_{x}}{n_{k}^{2}-K_{\perp}} \frac{\left(n_{k}^{2}-K_{\|}\right) \sin \theta^{\prime}}{\left(n_{k}^{2}-K_{\|}-\bar{K} \cos ^{2} \theta^{\prime}\right)}
\end{aligned}
$$

where $\mathbf{E}$ has the components $\left(E_{k}, E_{\theta^{\prime}}, E_{\phi^{\prime}}\right)$ in the spherical coordinate system defined by $\mathbf{k}$, and

$$
\bar{K}=K_{\perp}-K_{\|}+\frac{K_{x}^{2}}{n_{k}^{2}-K_{\perp}}
$$

\subsection{Plane wave dyadic}

In order to construct $\mathbf{E}$ (r) in Eq. (4) we need to express $\exp (i \mathbf{k}$. r) in the spherical coordinate system. Following [6], the plane wave dyadic is

$$
\begin{aligned}
I e^{i \mathbf{k} \cdot \mathbf{r}}=-4 \pi \sum_{n=0}^{\infty} \sum_{m=-n}^{n}(-1)^{m} i^{n}\left[\frac{i}{k} \overrightarrow{\mathrm{Y}}_{n-m}\left(\theta^{\prime}, \phi^{\prime}\right) \vec{l}_{n m}(k r, \theta, \phi)+\frac{1}{n(n+1)}\right. \\
\left.\left\{\vec{\Phi}_{n-m}\left(\theta^{\prime}, \phi^{\prime}\right) \vec{m}_{n m}(k r, \theta, \phi)+i \vec{\Psi}_{n-m}\left(\theta^{\prime}, \phi^{\prime}\right) \vec{n}_{n m}(k r, \theta, \phi)\right\}\right]
\end{aligned}
$$

where $\vec{l}_{l \jmath}, \vec{m}_{l \jmath}$, and $\vec{n}_{l \jmath}$ are vector harmonic functions of degree $\imath$ and order $\jmath$, and $\overrightarrow{\mathrm{Y}}_{l \jmath}, \vec{\Phi}_{\imath \jmath}$, and $\vec{\Psi}_{l \jmath}$ are vector spherical harmonics [6,7]. These are defined in the appendix section of this paper. We have used the arrows to indicate that these functions are vectors in order to avoid confusion with various labels and indices.

\subsection{Incoming plane wave}

The plane wave incident on the blob is expressed in spherical coordinates by taking the dot product of the electric field vector of the incoming wave with the dyadic in (13). Thus,

$$
\begin{aligned}
\mathbf{E}_{i n}= & \mathbf{E}_{\mathbf{0}} e^{i \mathbf{k}_{0} \cdot \mathbf{r}}=-4 \pi \sum_{n=0}^{\infty} \sum_{m=-n}^{n}(-1)^{m} i^{n} \mathcal{E}_{0}\left[\frac{i}{k_{0}} E_{0 k} Y_{n-m}\left(\theta_{0}, \phi_{0}\right) \vec{l}_{n m}\left(k_{0} r, \theta, \phi\right)+\right. \\
& \left.\frac{1}{n(n+1)}\left\{\vec{E}_{0}^{P} \cdot \vec{\Phi}_{n-m}\left(\theta_{0}, \phi_{0}\right) \vec{m}_{n m}\left(k_{0} r, \theta, \phi\right)+i \vec{E}_{0} \cdot \vec{\Psi}_{n-m}\left(\theta_{0}, \phi_{0}\right) \vec{n}_{n m}\left(k_{0} r, \theta, \phi\right)\right\}\right]
\end{aligned}
$$

where $\theta_{0}$ and $\phi_{0}$ are the propagation angles in spherical coordinates. Additionally,

$$
\mathbf{E}_{0}=\mathcal{E}_{0}\left(E_{o k} \hat{k}_{0}+E_{0 \theta} \hat{\theta}_{0}+E_{0 \phi} \hat{\phi}_{0}\right) \equiv \mathcal{E}_{0}\left(E_{o k} \hat{k}+\vec{E}_{0}^{P}\right)
$$

where $\mathcal{E}_{0}$ is the amplitude of the electric field with the polarizations as indicated above, and the unit vectors are along the appropriate directions. For the incoming wave we can set $\phi_{0}=0$ without any loss of generality. For a prescribed $\theta_{0}$ we obtain two values of $k_{0}$ from (9). By choosing the appropriate $k_{0}$ we obtain the corresponding polarization from (7). For example, if the incoming wave is the ordinary wave in the electron cyclotron range of frequencies then we choose the $k_{0}$ appropriate for this wave. The vector harmonic functions in the expression for the incoming wave include only the spherical Bessel functions of the first kind [8]. 


\subsection{Fields inside the blob and scattered fields}

The electric fields inside the blob and the scattered wave fields are obtained in exactly the same way. We express the Fourier transformed electric field as

$$
\mathbf{E}(\mathbf{k})=\mathcal{E}_{k}\left[E_{k}\left(k, \theta^{\prime}, \phi^{\prime}\right) \hat{k}+E_{\theta^{\prime}}\left(k, \theta^{\prime}, \phi^{\prime}\right) \hat{\theta}^{\prime}+E_{\phi^{\prime}}\left(k, \theta^{\prime}, \phi^{\prime}\right) \hat{\phi}^{\prime}\right] \equiv \mathcal{E}_{k}\left(E_{k} \hat{k}+\vec{E}_{k}^{P}\right)
$$

where $\mathcal{E}_{k}=\mathcal{E}_{k}\left(k, \theta^{\prime}, \phi^{\prime}\right)$ is the amplitude of the field. From the dispersion relation (9) we know that $k$ depends only on $\theta^{\prime}$ and is independent of $\phi^{\prime}$. Also, for each $\theta^{\prime}$ there are only two values of $k$. The electric field polarizations given by (7), and illustrated in (11) and (12), depend on $\theta^{\prime}$ and the two values of $k$. So the Fourier transformed electric field vector $\mathbf{E}(\mathbf{k})$ is a function of $\theta^{\prime}$ and $\phi^{\prime}$ and is given by

$$
\mathbf{E}(\mathbf{k})=\sum_{\ell=1}^{2} \delta\left(k-k_{\ell}\right) \mathcal{E}_{k \ell}\left(\theta^{\prime}, \phi^{\prime}\right)\left[E_{k \ell}\left(\theta^{\prime}\right) \hat{k}+E_{\theta^{\prime} \ell}\left(\theta^{\prime}\right) \hat{\theta}^{\prime}+E_{\phi^{\prime} \ell}\left(\theta^{\prime}\right) \hat{\phi}^{\prime}\right]
$$

where the summation is over the two normal modes of the plasma given by (9). The summation includes the amplitude of the electric field of each of the two modes and the corresponding polarization as indicated in (16). The $\delta$ functions arise because for each $\theta^{\prime}$ there are only two discrete values of $k$. Since $\mathcal{E}_{k \ell}\left(\theta^{\prime}, \phi^{\prime}\right)$ has to be a periodic function of $\theta^{\prime}$ and $\phi^{\prime}$, it can be expressed in terms of the spherical surface harmonics $Y_{n m}\left(\theta^{\prime}, \phi^{\prime}\right)$ which form a complete set (see the appendix). So the Fourier transformed electric field is

$$
\mathbf{E}(\mathbf{k})=\sum_{\ell=1}^{2} \sum_{n^{\prime}=0}^{\infty} \sum_{m^{\prime}=-n^{\prime}}^{n^{\prime}} \mathcal{E}_{\ell n^{\prime} m^{\prime}} Y_{n^{\prime} m^{\prime}}\left(\theta^{\prime}, \phi^{\prime}\right)\left[E_{k \ell}\left(\theta^{\prime}\right) \hat{k}+E_{\theta^{\prime} \ell}\left(\theta^{\prime}\right) \hat{\theta}^{\prime}+E_{\phi^{\prime} \ell}\left(\theta^{\prime}\right) \hat{\phi}^{\prime}\right]
$$

We substitute (18) along with the dyadic (13) into (4). The integral over $k$ and $\phi^{\prime}$ can be easily carried out so that the following expression for the electric field is obtained

$$
\begin{aligned}
\mathbf{E}(\mathbf{r})= & -8 \pi^{2} \sum_{\ell=1}^{2} \sum_{n=0}^{\infty} \sum_{n^{\prime}=0}^{\infty} \sum_{m=-n}^{n}(-1)^{m} i^{n} \mathcal{E}_{\ell n^{\prime} m} \int_{0}^{\pi} d \theta^{\prime} \sin \theta^{\prime} Y_{n^{\prime} m}\left(\theta^{\prime}, 0\right) k_{\ell}^{2} \\
& {\left[\frac{i}{k_{\ell}} E_{k \ell} Y_{n-m}\left(\theta^{\prime}, 0\right) \vec{l}_{n m}\left(k_{\ell} r, \theta, \phi\right)+\frac{1}{n(n+1)}\left\{\vec{E}_{k \ell}^{P}\left(\theta^{\prime}\right) . \vec{\Phi}_{n-m}\left(\theta^{\prime}, 0\right) \vec{m}_{n m}\left(k_{\ell} r, \theta, \phi\right)+\right.\right.} \\
& \left.\left.\overrightarrow{i E}_{k \ell}^{P}\left(\theta^{\prime}\right) . \vec{\Psi}_{n-m}\left(\theta^{\prime}, 0\right) \vec{n}_{n m}\left(k_{\ell} r, \theta, \phi\right)\right\}\right]
\end{aligned}
$$

The above expression has two important features. It shows that the scattered and blob fields include all the normal modes of the plasma, not just the normal mode corresponding to the incoming wave. The fields also include all possible directions of propagation. As noted above, the form of the electric field vectors is the same for the fields inside the blob and the scattered fields. The change in the densities between the plasma in the blob and the background plasma is implemented through different values of the wave number $k$ and the polarizations of the corresponding fields.

For the blob fields, the vector harmonic functions include the spherical Bessel functions of the first kind since we require the fields to be regular at the origin. For the scattered fields, the vector harmonic functions include Hankel functions of the first kind [8]. The choice is dictated by the requirement that the spatially asymptotic form of the scattered fields correspond to outgoing wave fields.

\section{Boundary conditions}

We assume that there is no free charge density or current density on the surface of the blob which separates the interior of the blob from the background plasma. Then the boundary conditions require 
the continuity of the following at the surface of the blob $r=a$ [9]

$$
\begin{aligned}
{\left[\hat{r} . \stackrel{\leftrightarrow}{K}_{b l} \cdot \mathbf{E}_{b l}\right]_{r=a} } & =\left[\hat{r} . \stackrel{\leftrightarrow}{K}_{p l} .\left(\mathbf{E}_{i n}+\mathbf{E}_{s c}\right)\right]_{r=a} \\
{\left[\hat{r} . \mathbf{B}_{b l}\right]_{r=a} } & =\left[\hat{r} .\left(\mathbf{B}_{i n}+\mathbf{B}_{s c}\right)\right]_{r=a} \\
{\left[\hat{r} \times \mathbf{E}_{b l}\right]_{r=a} } & =\left[\hat{r} \times\left(\mathbf{E}_{i n}+\mathbf{E}_{s c}\right)\right]_{r=a} \\
{\left[\hat{r} \times \mathbf{B}_{b l}\right]_{r=a} } & =\left[\hat{r} \times\left(\mathbf{B}_{i n}+\mathbf{B}_{s c}\right)\right]_{r=a}
\end{aligned}
$$

where $\stackrel{\leftrightarrow}{K}_{b l}$ and $\stackrel{\leftrightarrow}{K}_{p l}$ are the permittivity tensors for the plasma inside the blob and the background plasma, respectively, $\mathbf{E}_{b l}$ is the electric field inside the blob, and $\mathbf{E}_{s c}$ is the scattered wave electric field. $\mathbf{B}$ are the the magnetic fields corresponding to the waves indicated by the subscripts and are obtained from Faraday's equation

$$
\mathbf{B}=\frac{1}{i \omega} \nabla \times \mathbf{E}
$$

The conditions (20), (22), and (23) are obtained from Gauss', Faraday's, and Ampere's equations, respectively, while the condition (21) is from $\nabla . \mathbf{B}=0$.

It can be easily shown that of the six boundary conditions only four are independent. We choose the four boundary conditions to be those given by (22) and (23). These boundary conditions lead to equations which relate the coefficients $\mathcal{E}_{\ell n^{\prime} m}^{b l}$ and $\mathcal{E}_{\ell n^{\prime} m}^{s c}$ for the blob and scattered fields, respectively, to the incident field amplitude $\mathcal{E}_{0}$ in (14) and (15). Without loss of generality, we can set $\mathcal{E}_{0}=1$, which means that the fields inside the blob and the scattered fields are normalized to the amplitude of the electric field of the incoming wave. In principle, the number of equations and the unknowns $\left(\mathcal{E}_{\ell n^{\prime} m}^{b l}, \mathcal{E}_{\ell n^{\prime} m}^{s c}\right)$ are infinite. In practice, the number of equations and unknowns are limited by the order of the spherical Bessel functions. For a given argument of the Bessel functions, an upper limit on the order is reasonably well known since the amplitude of the Bessel functions decays rapidly as the order exceeds the argument [8]. In the computational results that are discussed below, we ensure that the results are numerically converged.

\section{Results and discussion}

We consider an ordinary wave in the EC frequency range incident on a blob of radius $2 \mathrm{~cm}$. The density of the background plasma, composed of deuterium ions and electrons, is taken to be $10^{19} \mathrm{~m}^{-3}$ and the ambient magnetic field is 4.13 Tesla. The EC frequency is $170 \mathrm{GHz}$ and the incoming ordinary wave is propagating in the $x-z$ plane at an angle of $\theta_{0}=88.6^{\circ}$ with respect to the direction of the magnetic field along the $z$ axis. Thus, $\phi_{0}=0$ and the free-space wavelength of the wave is $\lambda_{x} \approx 0.18 \mathrm{~cm}$ in the $x$-direction and $\lambda_{z} \approx 7.05 \mathrm{~cm}$ in the $z$-direction. The electric field vector of the ordinary wave is primarily along the $z$-direction.

In order to check our analytical formulation, as well as the corresponding numerical code, we first assume that the blob density is the same as the background density. Figures 1a and 1b show the phase of $E_{z}$, the component of the electric field vector along the $z$-direction, in the $x-z$ and $y-z$ planes, respectively. The white outline of the blob is as shown in Fig. 1a. The spacing of the peaks in the $\mathrm{x}$-direction in Fig. 1a corresponds to $\lambda_{x}$. Figure $1 \mathrm{~b}$ shows that the wavelength along the $z$-direction is approximately equal to $\lambda_{z}$ and the wave is uniform along the $y$-direction. The wave structure, as expected, is exactly the same inside the blob as outside and there is no discontinuity in the electric field across the blob boundary. So Figs. $1 \mathrm{a}$ and $1 \mathrm{~b}$ are just the pictorial representation of the ordinary plane wave. The phase of $E_{y}$, the $y$-component of the electric field, is exactly the same as shown in Figs. 1a and $1 \mathrm{~b}$. This is not surprising since the electric field vector is that of a pure plane wave. It is important to note that the entire analytical formalism described in the previous sections is used to obtain the results in Figs. 1a and 1b.

In the next set of figures we take the blob density to be $2 \times 10^{19} \mathrm{~m}^{-3}$, which is twice the background density. In Fig. 2 a we plot the phase of $E_{z}$ in the $x-z$ plane. The discontinuity in $E_{z}$ near the edge of the 


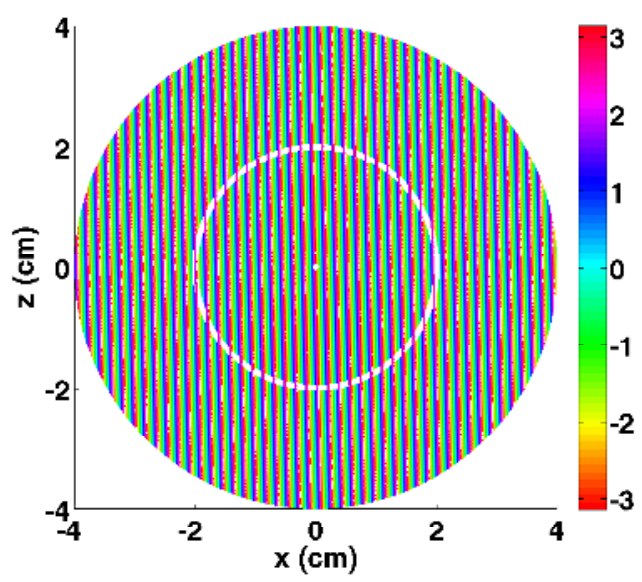

(a)

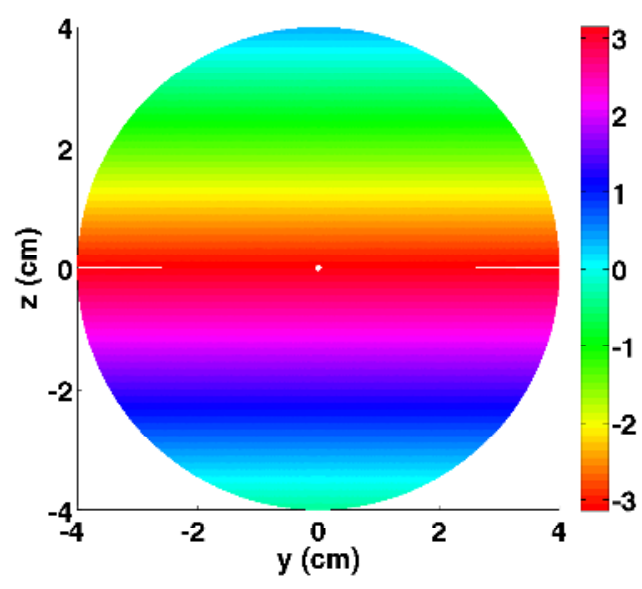

(b)

Fig. 1. Here we have assumed that the blob density is the same as the background density. The relevant parameters are as noted in the text. The incident ordinary wave is propagating from the left. (a) is a plot of the phase of $E_{z}$ in the $\mathrm{X}-\mathrm{z}$ plane, with the white outline of the blob; (b) is a plot of the phase of $\mathrm{E}_{z}$ in the $\mathrm{y}-\mathrm{z}$ plane.

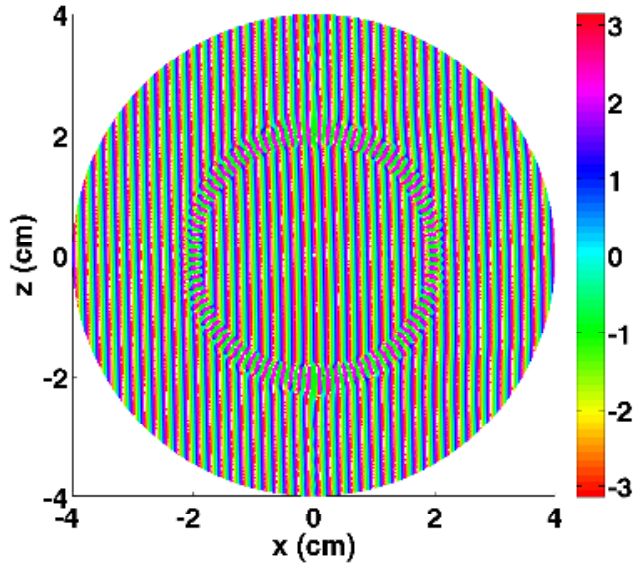

(a)

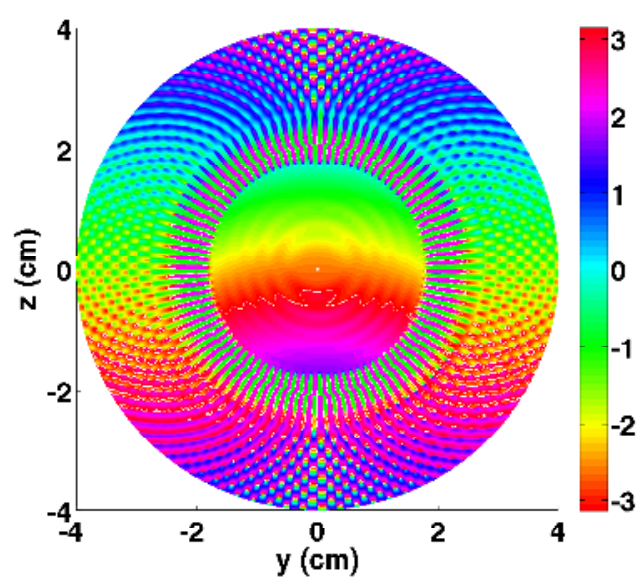

(b)

Fig. 2. The blob density is taken to be twice the background density. The rest of the parameters are the same as in Fig. 1. (a) is the phase of $E_{z}$ in the $x-z$ plane; (b) is the phase of $E_{y}$ in the $y-z$ plane.

blob, required by the boundary conditions, is quite evident. However, it is interesting to note that the phase remains almost similar to that in Fig. 1a in the regions away from the boundary layer. However, in the $y-z$ plane, the phase of $E_{y}$ in Fig. $2 \mathrm{~b}$ is quite different from that in Fig. 1b. The phase structure is an indication that the extraordinary wave is excited through the scattering process. The electric field of the extraordinary wave has a significant component along the $y$-direction.

In Figs. $3 \mathrm{a}$ and $3 \mathrm{~b}$ we plot, in the $x-z$ plane, the amplitude of the total electric field $|\mathbf{E}|$ inside and outside the blob. Figure 3a shows that the electric field amplitude is markedly enhanced in the region near the boundary of the blob. It is also evident that electric fields are propagating along the $\pm z$-directions which is the direction of the ambient magnetic field. If we suppress the large amplitude fields in the vicinity of the blob boundary, the structure of the scattered fields and the fields inside the blob become more evident. This is shown in Fig. $3 \mathrm{~b}$ where we note that most of the scattering is in the forward direction. There is very little backscatter. The incoming ordinary plane wave is incident on 


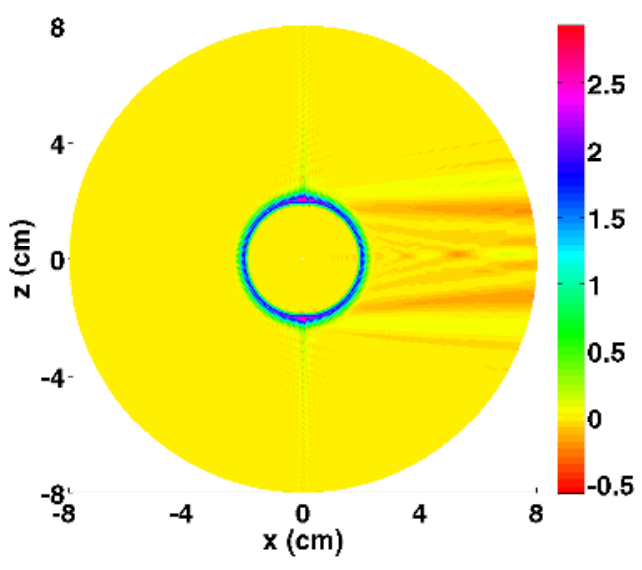

(a)

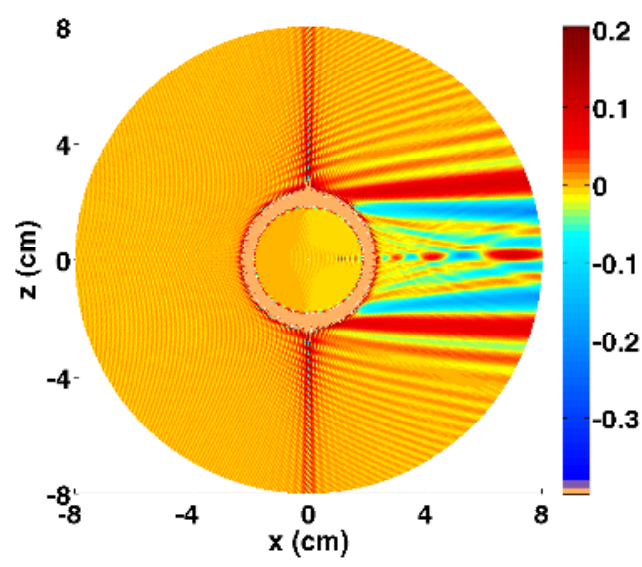

(b)

Fig. 3. These results are for the same parameters as in Fig. 2. (a) is a plot of the amplitude of the total electric field $|\mathbf{E}|$ in the $x-z$ plane; (b) is the same as (a) except that the high amplitude field in the vicinity of the surface of the blob has been suppressed. This is done in order to show the structure of the lower amplitude electric fields inside the blob and in the surrounding plasma region.

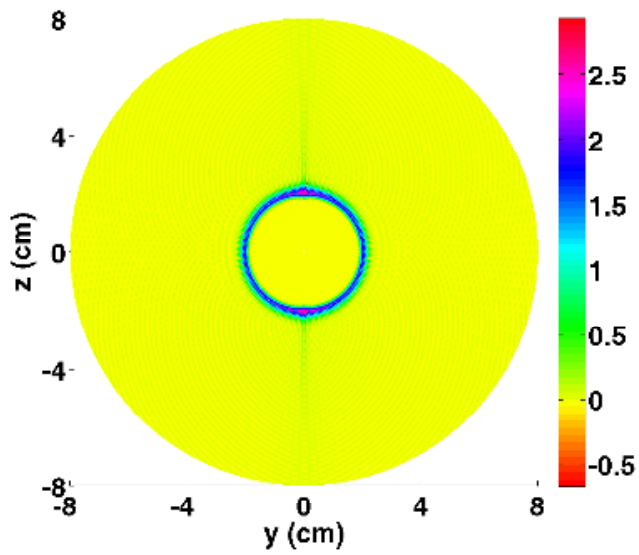

(a)

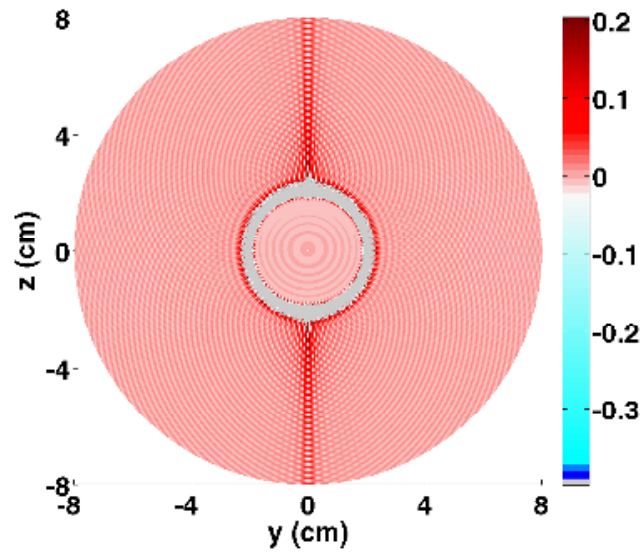

(b)

Fig. 4. This figure is the same as Fig. 3 except that the plots are in the $y-z$ plane. (a) is a plot of $|\mathbf{E}|$; (b) is the same as (a) except that the high amplitude electric field amplitudes in the vicinity of the surface of the blob have been suppressed.

the blob from the left. The spectrum of the wave propagating towards the core of the plasma after the interaction with a blob is quite broad. The electric fields of waves propagating along the $\pm z$-direction have significant amplitudes.

In Figs. $4 \mathrm{a}$ and $4 \mathrm{~b}$ we plot $|\mathbf{E}|$ in the $y-z$ plane. The field patterns inside and outside the blob are spatially structured and an indication of the extraordinary waves being excited by the interaction. The enhanced fields along the $\pm z$-directions point to the excitation of surface modes that are propagating along, or opposite, to the direction of the ambient magnetic field. 


\section{Conclusions}

We have developed a full-wave model for illustrating the effect of density blobs on the propagation of radio frequency waves. This study is quite relevant since the edge region of tokamak plasmas is dominated by fluctuations and blobs. The radio frequency waves have to interact with this active region as they propagate from the excitation structure toward the core of the plasma. The geometric optics analysis has shown that the interaction of electron cyclotron waves with a distribution of blobs can spatially deflect the wave in ITER-type plasmas [3]. Furthermore, there is a spreading of the spectrum of the waves which would lead to a broadened current profile. The full-wave scattering model is not limited by the restrictions inherent to the geometric optics approximation. This model applies to blobs with densities of arbitrary proportions relative to the background density. The full-wave model shows that electron cyclotron waves are diffracted by blobs and that the subsequent spectrum of the propagating waves is affected. Waves that are different from the incident waves are excited and the propagation of these waves is spatially spread out. The electric fields in the boundary layer surrounding the edge of the blob are enhanced and surface waves propagating along the direction of the ambient magnetic field are excited. A detailed quantitative analysis of the scattering will be published elsewhere.

\section{Acknowledgements}

We are grateful to Dr. Richard Garner for numerous informative and suggestive discussions. This work was supported by US DOE Grant number DE-FG02-91ER-54109, the European Fusion Program (Association EURATOM Hellenic Republic), and the Hellenic General Secretariat of Research and Technology.

\section{Appendix}

The vector harmonic functions and the vector spherical harmonics are discussed in [6,7]. We follow the notation used in [7]. The vector harmonic functions are given by

$$
\begin{aligned}
\vec{l}_{n m}(k r, \theta, \phi) & =\nabla \psi_{n m}(k r, \theta, \phi) \\
\vec{m}_{n m}(k r, \theta, \phi) & =-\mathbf{r} \times \nabla \psi_{n m}(k r, \theta, \phi) \\
\vec{n}_{n m}(k r, \theta, \phi) & =\frac{1}{k} \nabla \times \vec{m}_{n m}(k r, \theta, \phi)
\end{aligned}
$$

Here

$$
\psi_{n m}(k r, \theta, \phi)=z_{n}(k r) P_{n}^{m}(\cos \theta) e^{i m \phi}
$$

is the solution, in spherical coordinates, of the scalar Helmholtz equation, $P_{n}^{m}(\cos \theta)$ is the associated Legendre function of the first kind of degree $n$ and order $m$ [8], and $z_{n}$ is the spherical Bessel function of the first or the second kind, or the spherical Hankel function of the first or second kind.

The vector spherical harmonics are

$$
\begin{aligned}
& \vec{Y}_{n m}(\theta, \phi)=\hat{r} Y_{n m}(\theta, \phi) \\
& \vec{\Psi}_{n m}(\theta, \phi)=r \nabla Y_{n m}(\theta, \phi) \\
& \vec{\Phi}_{n m}(\theta, \phi)=\mathbf{r} \times \nabla Y_{n m}(\theta, \phi)
\end{aligned}
$$

where

$$
Y_{n m}(\theta, \phi)=\sqrt{\frac{(2 n+1)}{4 \pi} \frac{(n+m) !}{(n-m) !}} P_{n}^{m}(\cos \theta) e^{i m \phi}
$$

is the normalized surface harmonic of the first kind [8]. 


\section{References}

1. S. I. Krasheninnikov, Physics Letters A 283, (2001) 368-370.

2. S. J. Zweben, R. J. Maqueda, D. P. Stotler, A. Keesee, J. Boedo, C. E. Bush, S. M. Kaye, B. LeBlanc, J. L. Lowrance, V. J. Mastrocola, R. Maingi, N. Nishino, G. Renda, D. W. Swain, J. B.Wilgen, and the NSTX Team, Nuclear Fusion 44 (2004) 134-153.

3. K. Hizanidis, A. K. Ram, Y. Kominis, and C. Tsironis, Physics of Plasmas 17, (2010) 022505:1-10.

4. S. J. Zweben, J. A. Boedo, O. Grulke, C. Hidalgo, B. LaBombard, R. J. Maqueda, P. Scarin, and J. L. Terry, Plasma Physics and Controlled Fusion 49, (2007) S1-S23.

5. T. H. Stix, Waves in Plasmas (Springer, New York, 1992) Chapters 1 and 2.

6. J. A. Stratton, Electromagnetic Theory (McGraw Hill, New York, 1941) Chapter VI.

7. R. G. Barrera, G. A. Estévez, and J. Giraldo, European Journal of Physics 6, (1985) 287-294.

8. M. Abramowitz and I. A. Stegun, Handbook of Mathematical Functions (Dover, New York, 1972) Chapters 8 and 10.

9. D. J. Griffiths, Introduction to Electrodynamics (Pearson, New Jersey, 1999) Chapter 7. 